\title{
A Case Report of the Intravascular Fasciitis of a Neck Vein Mimicking Intravascular Tumorous Conditions
}

\author{
Ji-Hee Kang, MD, ${ }^{1}$ Dong-lk Kim, MD, PhD, ${ }^{2}$ Byung-Hoon Chung, MD, ${ }^{1}$ Seon-Hee Heo, MD, ${ }^{1}$ \\ and Yang-Jin Park, MD, PhD ${ }^{1}$
}

Intravascular fasciitis is a rare variant of nodular fasciitis, which can be easily misdiagnosed as a tumorous condition. We had a patient with an intravenous mass of a neck vein, and surgical excision was successful. Although all preoperative imaging studies and intraoperative pathologic reports suggested certain tumorous conditions as differential diagnosis results, the final diagnosis confirmed that it was an intravascular fasciitis based on its fibromixoid tissues with the proliferation of spindle cells and positive immunohistochemical staining for smooth muscle actin. Unless a physician has an insight of the disease or a suspicion to initiate running differential markers, it may be confused with other intravascular lesions and cause unnecessary radical surgery. Here we report our experience with a patient having this rare vascular disease.

Keywords: intravascular fasciitis, intravascular mass

\section{Introduction}

Intravascular fasciitis is a variant of nodular fasciitis, which is caused by a reactive intravascular proliferation of

${ }^{1}$ Division of Vascular Surgery, Department of Surgery, Samsung Medical Center, Sungkyunkwan University School of Medicine, Seoul, Korea

${ }^{2}$ Division of Vascular Surgery, Department of Surgery, Heart, Stroke and Vascular Institute, Samsung Medical Center, Sungkyunkwan University School of Medicine, Seoul, Korea

Received: May 8, 2018; Accepted: August 18, 2018

Corresponding author: Dong-Ik Kim, MD, PhD. Division of Vascular Surgery, Department of Surgery, Heart, Stroke and Vascular Institute, Samsung Medical Center, Sungkyunkwan University School of Medicine, Irwon-ro \#81, Gangnam-gu, Seoul 06351, Korea

Tel: +82-2-3410-3467, Fax: +82-2-3410-0040

E-mail: dongik.kim@samsung.com

(cc) BY-NC-SA (C2018 The Editorial Committee of Annals of Vascular Diseases. This article is distributed under the terms of the Creative Commons Attribution License, which permits use, distribution, and reproduction in any medium, provided the credit of the original work, a link to the license, and indication of any change are properly given, and the original work is not used for commercial purposes. Remixed or transformed contributions must be distributed under the same license as the original. myofibroblasts. This is most commonly found on the extremities, head, and neck as was the case in our patient. ${ }^{1)}$

We report the case of a 44-year-old female patient complaining about her left supraclavicular mass, which was later pathologically confirmed as a mixture of several cell components that was consistent with intravascular fasciitis. We performed several imaging examinations and surgically resected it. The objective of this report is to share our clinical experience of an intravenous mass mimicking a tumorous condition, such as leiomyoma.

\section{Case Report}

A 44-year-old woman visited our outpatient clinic presenting with a left supraclavicular mass, which was first palpated approximately 3 months ago. She has been on an antiviral medication for chronic hepatitis B; no other significant medical issues noted. The previous surgical history was cesarean section, twice. During physical examination, a well-defined soft mass was palpated right above the left supraclavicular area, which was an ovoid-shaped mass of approximately 40-mm length and was very close to the posterior side of the left clavicle.

Computed tomography showed a $41 \times 10 \mathrm{~mm}$-sized enlarged left clavicular intravenous structure with linear contrast filling inside (Fig. 1). Likewise, magnetic resonance imaging showed a longitudinal mass-like lesion of venous structure originating from the bifurcation of the brachiocephalic and internal jugular veins (Fig. 1). Intravenous spaces occupying lesions, such as thrombosis or tumor, are suggested to be differentiated by a radiologist. Duplex ultrasonography revealed the possibility of a connection between the mass and the left external jugular and subclavian veins. Because no definite malignant features were delineated on imaging studies but still could not be excluded, an ultrasonography-guided biopsy was performed. The pathologic report of the biopsy revealed that the mass contained fibrous tissues with chronic inflammation. Because of the patient's private circumstance, she revisited the clinic after 3 months of observation. Vague 


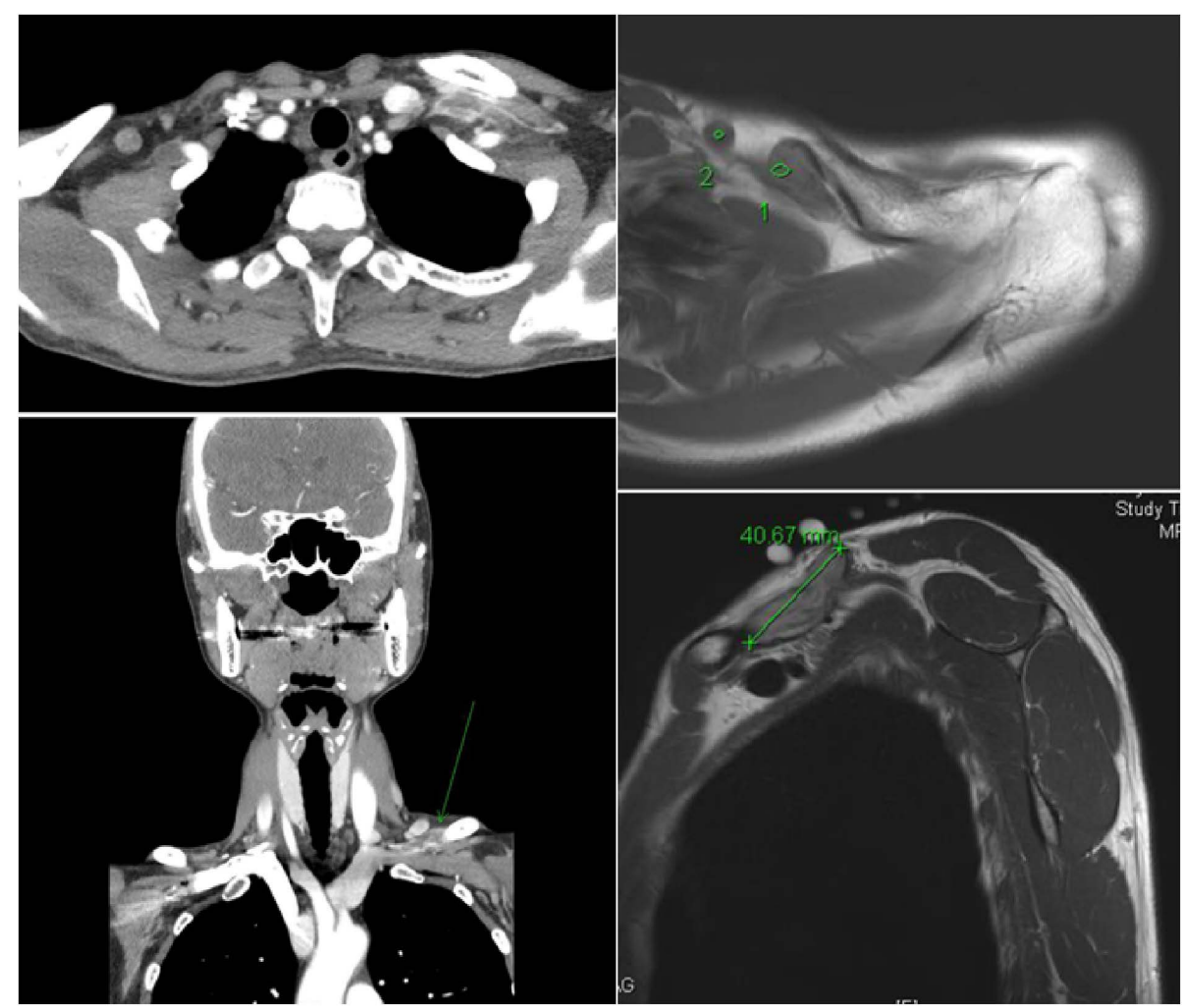

Fig. 1 Computed tomography showing an enlarged venous structure at the left supraclavicular area with linear contrast filling (left); magnetic resonance imaging showing a well-enhanced longitudinal mass-like lesion in the left supraclavicular fossa (right).

pain developed around the mass, and we scheduled a surgical excision. By that time, she did not present neurologic symptoms or arm swelling.

The surgery was initiated with a skin incision that was made along the left clavicle. For better exposure, an orthopedist joined the operation to transect the left clavicle. Next, the left subclavian and external jugular veins were isolated. In the operating field, a long ovoid well-circumscribed mass (Fig. 2) was transversely located between the left external jugular and the subclavian veins, with connections to adjacent veins as well as the external jugular vein. The proximal end was extended to the internal jugular vein but did not involve the intraluminal space. The external jugular vein and other connecting branches were ligated. Extravascular structures were not adhered to the mass. Considering a possible wide excision in case of malignancy, a piece of tissue was sent for a frozen pathology report.

Consequently, a pathologist reported that the mass was a tumor comprising spindle cells without definite malignant features. Possible diagnoses were leiomyoma, leiomyosarcoma, and less likely hemangioepithelioma. We then completed the surgery by performing just a mass excision without the need of a venous reconstruction. The left clavicle was also repaired in its original anatomic

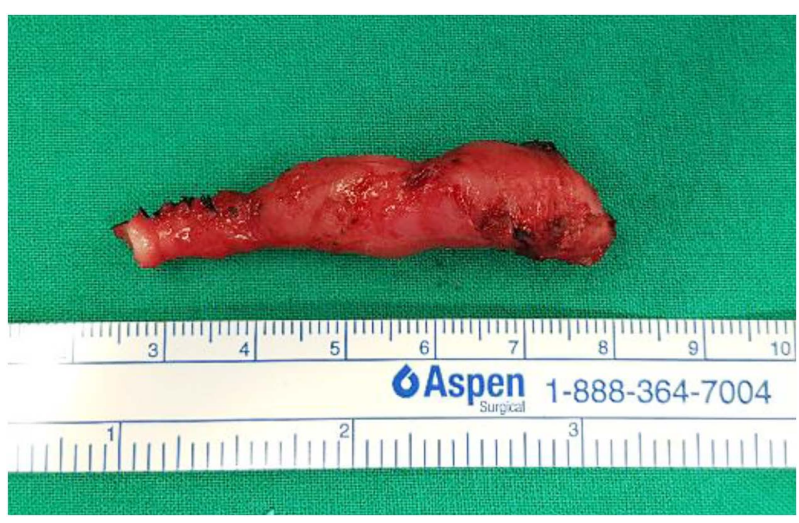

Fig. 2 Gross specimen showing a well-circumscribed soft ovoid mass.

space.

Pathologic examination was based on a microscopic investigation, which reported that the mass was a fibromyxoid tissue with a focal spindle cell proliferation and inflammation. Immunohistochemical stainings were conducted, in which the results were positive for smooth muscle actin and negative for S-100. Overall, the results were consistent with intravascular fasciitis (Fig. 3).

The patient recovered well and should be on an arm sling for clavicle stabilization. We also checked chest 


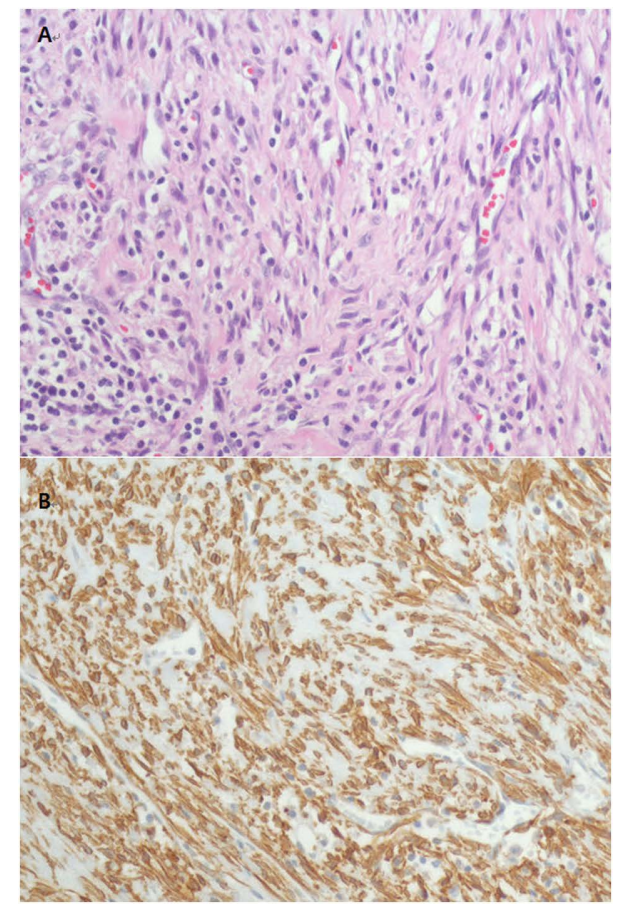

Fig. 3 (A) Photomicrographs revealing a focal infiltration of inflammatory cells (Hematoxylin \& eosin stain; magnification $\times 400$ ). (B) Smooth muscle actin (SMA) - positive myofibroblasts $(\times 400)$.

X-ray and confirmed no thoracic duct injury; thus, chylothorax developed. The patient was discharged on postoperative day 5 . She has been doing well on a follow-up visit after 1 month. The next follow-up will be 1 year later, and the patient will be under surveillance for any potential malignant behavior of this rare disease.

\section{Discussion}

A common reactive proliferation of soft tissue is known as a nodular fasciitis. Nodular fasciitis is a type of repair reaction that results in inflammation and, pathologically, the proliferation of fibroblasts and myofibroblasts. ${ }^{2)}$ Intravascular fasciitis (IF) is rarely found as a variant of nodular fasciitis, which was first described by Patchefsky and Enzinger. ${ }^{3)}$ IF can affect both arteries and veins. ${ }^{4)}$ Sizes of the involved vessels vary. ${ }^{3)}$ According to a previous case series, the majority of cases were found in the head and neck area, followed by the upper extremities, lower extremity, and trunk. ${ }^{5)}$ The commonly affected ones in the population are younger patients, with an average age of 24 years, although the disease itself is rarely found in the entire population. $\left.{ }^{6}\right)$ IF usually undergoes a clinically benign course, but few cases of recurrence after excision have been reported.6,7) Microscopically, IF is characterized by a spindle cell proliferation inside the lumen and can accompany organizing thrombi within the lesion. ${ }^{3)}$
The index of mitotic activity varies, but cytologic pleomorphism that can be found in a sarcoma is absent. ${ }^{8)}$ Immunohistochemistry showed that spindle cells were positive for smooth muscle actin but negative for keratin, S100, and desmin, which confirmed the myofibroblastic differentiation seen in our patient. However, the typical spindle-shaped myofibroblastic cells can still be misinterpreted as a sarcoma in which the myxoid and fibrous phases may be overinterpreted as malignancies. ${ }^{2}$ Without immunohistochemical staining, the intraoperative pathologic report from frozen sections might not be enough to make a diagnosis of IF. Other than the above-stated immunohistochemistry markers, spindle cells of nodular fasciitis contain vimentin and muscle-specific actin along with smooth muscle-specific actin. ${ }^{9)}$

Intravascular leiomyoma, which was initially suspected, has a histological similarity because it is characterized by the proliferation of smooth muscle cells. However, it is usually found in women who underwent hysterectomy due to uterine myoma. The proliferation of smooth muscle cells usually arises from the intrauterine venules and/or the myometrium. ${ }^{10)}$ Its clinical manifestation shows progression to involve the iliac veins, the inferior vena cava, and even the right atrium. ${ }^{11)} \mathrm{A}$ complete history taking can provide clues. Pathologically, this tumor contains endothelial cells that can be positive for CD31, CD34, vimentin, and smooth muscle actin. ${ }^{11}$

Several risk factors of IF have been proposed, including trauma and thrombosis, but the exact mechanism and causative factors remain unknown and should be investigated in the future. There are no established imaging criteria for an accurate diagnosis of IF. Only if a diagnosis of IF is pathologically suspected, appropriate differential markers should be run. ${ }^{7)}$ Otherwise, IF can be confused with other intravascular lesions, as was the case in our patient.

\section{Conclusion}

By reporting this rare disease, which is easily misdiagnosed as other tumorous conditions, we provide insights regarding IF, which should be considered whenever uncertain intravascular nodules without definite malignant features are encountered.

\section{Disclosure Statement}

All authors have no conflict of interest.

\section{Author Contributions}

Study conception: DIK, JHK

Data collection: DIK, JHK

Analysis: not applicable for a case report 
Investigation: DIK, JHK

Writing: JHK

Funding acquisition: not applicable

Critical review and revision: all authors

Final approval of the article: all authors

Accountability for all aspects of the work: all authors

\section{References}

1) Takahashi K, Yanagi T, Imafuku K, et al. Ultrasonographic features of intravascular fasciitis: case report and review of the literature. J Eur Acad Dermatol Venereol 2017; 31: e4579.

2) Forcucci JA, Bruner ET, Smith MT. Benign soft tissue lesions that may mimic malignancy. Semin Diagn Pathol 2016; 33 : 50-9.

3) Sugaya M, Tamaki K. Does thrombosis cause intravascular fasciitis? Acta Derm Venereol 2007; 87: 369-70.

4) Wang L, Wang G, Wang L, et al. Myxoid intravascular fasciitis. J Cutan Pathol 2011; 38: 63-6.

5) Chi AC, Dunlap WS, Richardson MS, et al. Intravascular fas- ciitis: report of an intraoral case and review of the literature. Head Neck Pathol 2012; 6: 140-5.

6) Samaratunga H, Searle J, O'Loughlin B. Intravascular fasciitis: a case report and review of the literature. Pathology 1996; 28: 8-11.

7) Ito M, Matsunaga K, Sano K, et al. Intravascular fasciitis of the forearm vein: a case report with immunohistochemical characterization. Pathol Int 1999; 49: 175-9.

8) Reiser V, Alterman M, Shlomi B, et al. Oral intravascular fasciitis: a rare maxillofacial lesion. Oral Surg Oral Med Oral Pathol Oral Radiol 2012; 114: e40-4.

9) Borumandi F, Cascarini L, Mallawaarachchi R, et al. The chameleon in the neck: Nodular fasciitis mimicking malignant neck mass of unknown primary. Int J Surg Case Rep 2012; 3: 501-3.

10) Morales MM, Anacleto A, Leal JC, et al. Intravascular leiomyoma with heart extension. Clinics (São Paulo) 2012; 67: 83-7.

11) Zhang Y, Clark LH, Sheng XG, et al. Successful en bloc venous resection with reconstruction and subsequent radiotherapy for 2 consecutive recurrences of intravenous leiomyoma-a case report. BMC Cancer 2016; 16: 6. 